\title{
NLRP3 inflammasome ignites podocyte dysfunction
}

the NLRP3
inflammasome
was found to
be activated
in podocytes
from biopsy
and urine
samples from
patients
with lupus
nephritis

Activation of the NLRP3 inflammasome results in podocyte injury and proteinuria in lupus nephritis, according to a new study. "The most significant finding in this project is that podocytes are active participants in the pathogenesis of lupus nephritis," says Niansheng Yang, corresponding author of the study.

Podocytes are highly specialized cells surrounding the glomerular capillaries and have a major role in blood filtration in the kidneys. Podocyte dysfunction is known to be involved in the pathogenesis of lupus nephritis, but the molecular mechanisms

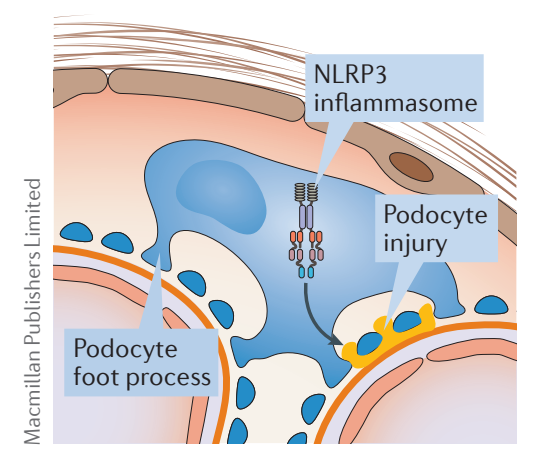

underlying the injury of these cells have not yet been elucidated. In this study, Yang and colleagues investigated the role of the NLRP3 inflammasome, a molecular complex of the innate immune system that activates caspase 1 and leads to the production of pro-inflammatory cytokines such as IL- $1 \beta$.

The investigators showed that the protein expression levels of NLRP3 and IL- $1 \beta$ were higher in NZM2328 lupus-prone mice with severe proteinuria than in control NZM2328 mice without proteinuria. Furthermore, the levels of active caspase 1 in podocytes from mice with proteinuria were higher than in those from control mice. Consistent with these findings, the NLRP3 inflammasome was found to be activated in podocytes from biopsy and urine samples from patients with lupus nephritis, but not in those from healthy donors.

To further confirm the role of NLRP3 in vivo, Yang and colleagues treated NZM2328 mice with the selective NLRP3 inhibitor MCC950.
Treatment with MCC950 significantly reduced the levels of IL- $1 \beta$ in the kidneys and inhibited the activation of caspase 1 in glomerular podocytes, as compared with mice treated with vehicle. In addition, treatment with MCC950 reduced the incidence of severe proteinuria in NZM2328 mice compared with vehicle-treated mice, which was associated with an attenuation of podocyte foot process effacement and a reduction of renal lesions.

These findings indicate that the NLRP3 inflammasome is activated in podocytes and contributes to the pathogenesis of lupus nephritis. Yang remarks that their basic findings provide impetus for further research on the interaction between podocytes and inflammatory cells, which might help to identify novel targets for therapy.

Dario Ummarino

ORIGINAL ARTICLE Fu, R. et al. Podocyte activation of NLRP3 inflammasomes contributes to the development of proteinuria in lupus nephritis. Arthritis Rheumatol http://dx.doi.org/10.1002/ art.40155 (2017) 